Final Report on Subcontract B591217: Multigrid Methods for Systems of PDEs

J. Xu, J. J. Brannick, L. Zikatanov

October 27, 2011 
This document was prepared as an account of work sponsored by an agency of the United States government. Neither the United States government nor Lawrence Livermore National Security, LLC, nor any of their employees makes any warranty, expressed or implied, or assumes any legal liability or responsibility for the accuracy, completeness, or usefulness of any information, apparatus, product, or process disclosed, or represents that its use would not infringe privately owned rights. Reference herein to any specific commercial product, process, or service by trade name, trademark, manufacturer, or otherwise does not necessarily constitute or imply its endorsement, recommendation, or favoring by the United States government or Lawrence Livermore National Security, LLC. The views and opinions of authors expressed herein do not necessarily state or reflect those of the United States government or Lawrence Livermore National Security, LLC, and shall not be used for advertising or product endorsement purposes.

This work performed under the auspices of the U.S. Department of Energy by Lawrence Livermore National Laboratory under Contract DE-AC52-07NA27344. 


\title{
Final Report on Subcontract B591217: Multigrid Methods for Systems of PDEs
}

\author{
June 1, 2010 - May 31, 2011 \\ PI: Jinchao Xu, CoPIs: James Brannick and Ludmil Zikatanov
}

Progress has been made in several of the proposed areas of study, as summarized below.

\section{Compatible Relaxation}

The project team has continued with work on developing Compatible Relaxation coarsening algorithms for both Classical and Aggregation AMG. Of particular interest here is development of a sharp compatible relaxation iteration, in terms of its' ability to estimate the performance of the resulting two-level algorithm based on the given set of coarse degrees of freedom. Towards this end several variants of habituated CR have been developed and implemented with Falgout, showing promising initial results. The results indicate that while the given Habituated CR iterations better predict two-level convergence, for some problems the spectral radius of the $\mathrm{CR}$ error propagation matrix does not provide an upper bound of the two-grid method's convergence. This in turn makes it difficult to use these schemes as tools to measure the quality of the coarse grid. Understanding this behavior is a topic of current research as is the development of a variant in Hypre (with Falgout and Yang). Using these new forms of CR iterations to define a candidate set measure for selecting coarse degrees of is another area we have made progress in.

Improving aggregation-based MG solver performance -- Variable cycling.

We have developed a general theoretical framework for defining variable (adaptive multilevel iteration) cycles in the aggregation-based MG setting for HPD systems. The theory provides an estimate that can be used to define variable cycles for different choices of aggregation techniques. We have confirmed the sharpness of the derived estimate through extensive tests, showing that for plain aggregation stability and hence optimality can be recovered using the given estimates. Our current work focuses on extending these techniques to non-Hermitian systems, for example, those arising in lattice QCD.

We have also derived a new commuting diagram relating the stability of Aggregation-based interpolation and an 12 estimate of a certain projection, which can be computed efficiently. The estimate has been further used to develop an AMG strength of connection measure for forming sets of aggregates. The approach effectively treats grid and non-grid aligned anisotropic problems.

Additionally, we have developed a new polynomial approximation to the matrix inverse and shown that by using this approximation it is possible to construct AMG smoothers with proven smoothing rates for SPD systems. We have extended this approach to non 
HPD systems as well as a Chebychev based alternative. Both have been tested extensively for LQCD systems and their performance is similar for low-degree approximations.

\section{FOSLS for LQCD.}

The project continued to develop First Order System Least Squares (FOSLS) discretizations of the Schwinger operator of QED and other QCD models. The standard FOSLS discretization directly forms the normal equations of the model problem in the continuum and then discretizes the resulting PDE using bilinear and Nedelec finite elements. The FOSLS methodology is beneficial from a physical perspective because it naturally avoids the problem of species-doubling without adding artificial diffusion terms that break chiral symmetry.

We have now successfully implemented and tested the FOSLS formulation for the $4 d$ QCD model with $U(1)$ gauge field and our numerical experiments show that the linear systems resulting from this discretization can be solved very effectively using standard AMG techniques, such as classical AMG or smoothed aggregation multigrid. Solver performance remains stable for both larger lattice sizes and more disordered gauge fields.

\section{Auxiliary Space Preconditioners.}

We conducted numerical tests that demonstrate the FOSLS formulation gives a good approximation to the low eigenvalues of the Dirac equation in the continuum, from which it follows that it should provide a good preconditioner, at least on the low modes of any suitable discretization. We have implemented a defect-correction type method based on this idea and carried out extensive tests for the 2d Schwinger model of QED. In the free case, the method exhibits optimal performance, however, in the gauged case, it still does not scale with increasing problem size or varying masses. Our tests indicate that the cause of the suboptimal performance in the gauged case is closely related to the addition of the WIlson like term. In particular, in the additional Wilson term pollutes the eigenpairs of the Wilson-Dirac system and thus the FOSLS formulation which accurately approximates the eigenpairs of the continuous Dirac operator is not effective as a preconditioner. Currently we succeeded in applying the defect correction scheme to other (more accurate) discretizations, for example the Overlap operator. Our initial results are promising.

\section{Bootstrap Algebraic Multigrid.}

We developed an adaptive Bootstrap AMG (BAMG) solver for Hermitian and nonHermitain matrix systems. The approach employs an adaptive setup algorithm to compute interpolation, based on the notions of compatible relaxation (CR) and least squares fitting of test vectors, i.e, a set of vectors computed adaptively. This is an alternative, and potentially far less expensive approach to our adaptive aggregationbased setup algorithm described in items 1 and 2 above. The adaptive aggregationbased setup algorithm generates the prototypes used in defining Multigrid interpolation sequentially, which requires recomputing the MG hierarchy at each adaptive step. In contrast, our BAMG adaptive aggregation-based setup, computes interpolation in a single (or few) adaptive step(s) using as a main tool an adaptive multi-level eigensolver. The BAMG approach has been shown to yield a solver whose performance is similar to that of the aggregation based scheme. Current work focuses on extending the BAMG 
solver nonsymmetric graph Laplacian problems and LQCD systems.

Practical Applications of AMG and Fast Auxiliary Space Preconditioners.

Efficiency of AMG methods for problems from complicated applications is very limited. On the other hand, AMG methods can play a crucial role in the Fast Auxiliary Space Preconditioning (FASP) methods to achieve nearly optimal complexity for a large class of problems, by making use of the geometric and analytic information of the underlying problems as much as possible. We have applied this philosophy in problems like underground water flow in porous media, petroleum reservoir simulation with traditional water/gas flooding as well as polymer flooding. The nature of these physical systems, like heterogeneity, nonlocal connections due to faults and different behavior of equations in the system, presents challenges to algebraic solvers. We found that AMG methods (with some proper tuning) used in the FASP framework still show promising features.

\section{$\underline{\text { References }}$}

1. J. Brannick, Y. Chen, J. Kraus, and L. Zikatanov, An Algebraic Multilevel Method for the Graph Laplacian based on Matching of Graphs, in preparation.

2. J. Brannick, and R. Falgout, Compatible relaxation and coarsening in algebraic multigrid, SIAM Journal of Scientific Computing, 32:3 (2010), pp. 1393-1416. Also available as LLNL technical report: LLNL-JRNL-417122.

3. A. Brandt, J. Brannick, K. Kahl, I. Livshits, Bootstrap AMG, SIAM Journal of Scientific Computing, 33:2 (2011).

4. R. Babich, J. Brannick, R. C. Brower, M. A. Clark, T. A. Manteuffel, S. F. McCormick, J. C. Osborn, C. Rebbi, Adaptive multigrid algorithm for the lattice Wilson-Dirac operator, Phys. Rev. Lett., to appear. Also available as arXiv:1005.3043v2 [hep-lat].

5. J. Brannick, C. Ketelsen, T. Manteuffel, and S. McCormick, Least squares finite element methods for Quantum Electrodynamics, SIAM J. Sci. Comp., 32(1) 398--417 (2010).

6. R. Cheng, X. Huang, S. Shu, J. Xu, C.-S. Zhang, S. Zhang, Z. Zhou, An energy-minimization based algebraic multigrid method with applications in subsurface water simulation, Journal of Numerical Analysis and Modelling, Submitted (2010).

7. J. Xu, Fast Poisson-Based Solvers for Linear and Nonlinear PDEs, Proceedings of the International Congress of Mathematicians Hyderabad, India, 2010. 